\title{
Probiotics for Preterm Infants - The Story Searching for an End
}

\author{
SAnjay Patole \\ Centre for Neonatal Research and Education, University of Western Australia, Perth, Australia. \\ sanjay.patole@health.wa.gov.au
}

A

dvances in neonatal intensive care have increased the survival rates of extremely preterm infants. Necrotizing enterocolitis (NEC), late onset sepsis (LOS) and suboptimal nutrition raise the risk of death and long-term neurodevelopmental impairment (NDI) in this population. Improving survival without long-term NDI in extremely preterm infants is a priority. Interventions that optimize enteral nutrition while decreasing the risk of NEC and LOS are therefore urgently needed for preterms, especially extremely preterm infants.

Probiotics are live microorganisms that provide benefit to the host, when administered in an adequate amount. Data from systematic reviews of randomized controlled trials (RCTs) and non-RCTs confirm that probiotics significantly reduce the risk of $\geq$ Stage II NEC, all-cause mortality and LOS, and improve feed tolerance in preterm very low birth weight (VLBW) infants [1]. No other intervention in neonatal medicine comes close to probiotics in terms of the effect size for NEC and mortality, and supporting evidence from research and clinical practice [2].

Careful scrutiny of the literature reveals only few issues that are sustaining the debate about probiotics for preterm infants. These include the view that probiotic effects are strain-specific (i.e. all meta-analyses in this field are null and void!), the difficulty in accessing safe and clinical proven probiotic products, and the risk of probiotic sepsis. Almost all other issues, including 'We don't know how probiotics work', 'Probiotics are not needed if the infant is fed breastmilk - the nature's synbiotic, or when the baseline incidence of NEC is low', 'The PIPs trial showed that not all probiotic strains are effective, and challenged the previous meta-analyses', 'There is not enough data on extremely low birth weight (ELBW) infants, and probiotics may not have significant benefits in this population', have been addressed over the last few years [3-7]. Probiotic sepsis is easy to treat compared to sepsis by other potentially disastrous (e.g. gram negative) organisms. If contamination of the control arm underestimates the true effects of probiotic in RCTs, the results of the cluster RCT by Totsu, et al. [8] are reassuring. Substantial evidence indicates that probiotic supplementation also benefits ELBW infants [9].

In this issue of Indian Pediatrics, Shashidhar, et al. [10] report the effect of probiotic supplementation on the time to full feeds (Primary outcome) in preterm VLBW (Birth weight: 750-1499 g) infants randomly allocated to receive either breast milk with a multi-strain probiotic (Lactobacillus acidophilus, Lactobacillus rhamnosus, Bifidobacterium longum and Saccharomyces boulardii once a day; dose: $1.25 \times 10^{9} \mathrm{CFU}$ ) from start of feeds till discharge (Probiotic group: $n=52$ ) or only breast milk (Control group: $n=52$ ). The mean (SD) time to full feeds $(150 \mathrm{~mL} / \mathrm{kg} /$ day $)$ was not significantly different between the probiotic $v s$ no probiotic group [11.2 (8.3) vs 12.7 (8.9) days, $P=0.4]$. There was a trend towards lower NEC in the probiotic group ( $4 \%$ vs $12 \%)$. The limitations of this RCT include unrealistic desired effect for estimating sample size, higher frequency of ELBW infants and caesarean deliveries in the control group, and possibility of cross contamination [10]. The report raises few important questions: the utility of small trials, safety of using over the counter probiotics, and the difficulty in balancing the ethics, economics, and politics about probiotics for preterm neonates, especially in resourcelimited set-ups.

Reproducibility is at the core of science. Unexpected contradictory results of the recent RCT of antenatal glucocorticoids in India is a good example of how adopting an intervention in a different set-up without confirming its benefits-risks, is risky [11]. However, overcoming the difficulties in conducting adequately powered RCTs in resource-limited set-ups, and interpreting the results of small trials is not easy. Small trials are prone to give uncertain, false positive or false negative results, and without adequate resources the risk of bias in various aspects of methodology is high. Metaanalysis of RCTs specifically from such set ups is an option. Apologies to those who are tired of meta-analyses in this field or have firm belief in strain-specific effects of probiotics! However, there is hope for a change in 
opinion. Based on the consistently decreased risk of NEC in RCTs using variable probiotic regimens, experts suggest that it is time we accept that commonly used probiotic strains share pathways of benefits providing 'non-specific' protection [12,13]. As for the difficulties in accessing safe and clinically proven products, an urgent collaboration between all stakeholders is required to develop and test indigenous high quality probiotics and improve the process of importing such products. The goal is clear. Can India take the initiative?

Funding: None; Competing interests: None stated.

\section{REFERENCES}

1. Dermyshi E, Wang Y, Yan C, Hong W, Qiu G, Gong X, et al. The "Golden Age" of probiotics: A systematic review and meta-analysis of randomized and observational studies in preterm infants. Neonatology. 2017;112:9-23.

2. Ofek Shlomai N, Deshpande G, Rao S, Patole S. Probiotics for preterm neonates: what will it take to change clinical practice? Neonatology. 2014;105:64-70.

3. Jacobs SE, Tobin JM, Opie GF, Donath S, Tabrizi SN, Pirotta M, et al,; ProPrems Study Group. Probiotic effects on late-onset sepsis in very preterm infants: a randomized controlled trial. Pediatrics. 2013;132:1055-62.

4. Samuels N, van de Graaf R, Been JV, de Jonge RC, Hanff LM, Wijnen RM, et al. Necrotising enterocolitis and mortality in preterm infants after introduction of probiotics: A quasi-experimental study. Sci Rep. 2016;6:31643.

5. Repa A, Thanhaeuser M, Endress D, Weber M, Kreiss1 A, Binder C, et al. Probiotics (Lactobacillus acidophilus and Bifidobacterium infantis) prevent NEC in VLBW infants fed breast milk but not formula [Corrected]. Pediatr Res.
2015;77:381-8.

6. Karthikeyan G, Bhat BV. The PiPS (Probiotics in Preterm Infants Study) trial - Controlling the confounding factor of cross-contamination unveils significant benefits. Indian Pediatr. 2017;54:162.

7. Deshpande G, Rao S, Athalye-Jape G, Conway P, Patole S. Probiotics in very preterm infants: the PiPS trial. Lancet. 2016;388:655.

8. Totsu S, Yamasaki C, Terahara M, Uchiyama A, Kusuda S; Probiotics Study Group in Japan. Bifidobacterium and enteral feeding in preterm infants: cluster-randomized trial. Pediatr Int. 2014;56:714-9.

9. Denkel LA, Schwab F, Garten L, Geffers C, Gastmeier P, Piening B. Protective effect of dual-strain probiotics in preterm infants: A multi-center time series analysis. PLoS One. 2016;11:e0158136.

10. Shashidhar A, Suman Rao PN, Nesargi S, Bhat S, Chandrakala BS. Probiotics for promoting feed tolerance in very low birth weight neonates - A randomized controlled trial. Indian Pediatr. 2017;54:363-7.

11. Althabe F, Belizán JM, McClure EM, Hemingway-Foday $\mathrm{J}$, Berrueta M, Mazzoni A, et al. A population-based, multifaceted strategy to implement antenatal corticosteroid treatment versus standard care for the reduction of neonatal mortality due to preterm birth in low-income and middleincome countries: the ACT cluster-randomised trial. Lancet. 2015;385:629-39.

12. California Dairy Research Foundation. Sanders ME. Is it time to consider generic probiotic effects? Available from: http://cdrf.org/2013/03/01/is-it-time-to-consider-genericprobiotic-effects/. Accessed March 30, 2017.

13. GanguliK, Walker WA. Probiotics in the prevention of necrotizing enterocolitis. J Clin Gastroenterol. 2011; 45: S133-S138. 